Far Eastern Entomologist

\begin{tabular}{lll}
\hline Number 423: 5-8 & ISSN 1026-051X (print edition) & January 2021 \\
\hline
\end{tabular}

https://doi.org/10.25221/fee.423.2

http://zoobank.org/References/8877936F-7EFE-45CE-8803-D44446BAB888

\title{
A LIST OF PLUME MOTHS (LEPIDOPTERA: PTEROPHORIDAE) OF THE FAUNA OF PAKISTAN
}

\author{
P. Ya. Ustjuzhanin ${ }^{1,2, *)}$, V. N. Kovtunovich ${ }^{3)}$, V. V. Sinyaev ${ }^{4)}$, \\ S. I. Evdoshenko ${ }^{5)}$, A. N. Streltzov6)
}

1) Altai State University, Lenina 61, Barnaul, 656049, Russia. *Corresponding author, E-mail:petrust@mail.ru

2) Biological Institute, Tomsk State University, Lenina 36, Tomsk, 634050, Russia.

3) Moscow Society of Nature Explorers, Bolshaya Nikitskaya 2, Moscow, 125009, Russia.E-mail:vasko-69@mail.ru

4) Autonomous non-profit organization "Eco-design studio", Yuzhnoportovy proezd 15174, Moscow, 115432, Russia.E-mail: fieldcollector@inbox.ru

5) Belarus Entomolgical Society, Minsk,220072,Belarus.E-mail:daph@list.ru

6) Herzen State Pedagogical University of Russia, 48, Moika Emb., Saint-Petersburg, 191186, Russia.E-mail: streltzov@mail.ru

Summary. An annotated list of 19 species of Pterophoridae recorded from Pakistan is given. Five species are new to this country, namely Platyptilia avicennai Ustjuzhanin et Kovtunovich, 2016, Cnaemidophorus rhododactylus (Denis et Schiffermüller, 1775), Stenoptilia stigmatodactyla (Zeller, 1852), Hellinsia pectodactyla (Staudinger, 1859), and Amblyptilia sp. A new synonymy is established: Exelastis ebalensis $($ Rebel, 1907) $=$ Karachia xylochromella Amsel, 1968, syn. n.

Key words: Pterophoridae, plume moths, new synonymy, fauna, new records, Pakistan.

П. Я. Устюжанин, В. Н. Ковтунович, В. В. Синяев, С. И. Евдошенко, А. Н. Стрельцов. Список пальцекрылок (Lepidoptera: Pterophoridae) фауны Пакистана // Дальневосточный энтомолог. 2021. N 423. C. 5-8.

Резюме. Приводится список достоверно указанных для Пакистана 19 видов пальцекрылок (Pterophoridae). Впервые для страны отмечены 5 видов: Platyptilia avicennai Ustjuzhanin et Kovtunovich, 2016, Cnaemidophorus rhododactylus (Denis et Schiffermüller, 1775), Stenoptilia stigmatodactyla (Zeller, 1852), Hellinsia pectodactyla (Staudinger, 1859) и Amblyptilia sp. Установлена новая синонимия: Exelastis ebalensis $($ Rebel, 1907) $=$ Karachia xylochromella Amsel, 1968, syn. n.

\section{INTRODUCTION}

The territory of Pakistan is rich in various natural landscapes (from deserts and semideserts to highlands). The fauna is represented by Indo-African, Central Asian and Mediterranean species. The plume moths (Pterophoridae) of Pakistan are studied very poorly and fragmentary. Until present, the only 14 species are known from Pakistan (Amsel, 1968; Gibeaux, 1992; Arenberger, 1999b; Gielis, 2003). Present paper is based on the material collected 
in the northern part of this country in the provinces Gilgit Baltistan and Nord West Frontier by Viktor Sinyaev and Sergey Evdoshenko in 2019. As a result, five species are recorded for Pakistan the first time. List of plume moths of Pakistan is given below.

\section{LIST OF THE SPECIES}

Agdistis arabica Amsel, 1958

DISTRIBUTION. Pakistan: Karachi (Arenberger, 1995). - Tunisia, Egypt, Israel, Sudan, Yemen, Saudi Arabia, Oman, Bahrain, Iran, Somalia.

Agdistis nanodes Meyrick, 1906

DISTRIBUTION. Pakistan: Karachi (Arenberger, 1995). - Egypt, Saudi Arabia, Yemen, Bahrain, Oman, Iran, Sri Lanka.

\section{Agdistis tamaricis Zeller, 1847}

DISTRIBUTION. Pakistan : Karachi (Arenberger, 1995). - Europe, Turkmenistan, Kazakhstan, Kyrgyzstan, Tajikistan, Uzbekistan, Iran, Afghanistan, Mongolia, China (Tianjin, Hebei, Inner Mongolia, Shanghai, Shandong, Shaanxi, Gansu, Ningxia, Taiwan), Liberia, Southern Africa, Mauritania.

\section{Platyptilia avicennai Ustjuzhanin et Kovtunovich, 2016}

MATERIAL EXAMINED. Pakistan: an administrative territory Gilgit Baltistan, Astor River, Chilam, $35^{\circ} 08^{\prime} \mathrm{N}, 7^{\circ} 02^{\prime} \mathrm{E}, \mathrm{h}=3050 \mathrm{~m}, 1-4$.VIII 2019, 2 今े, 1 ㅇ, S. Evdoshenko \& V. Siniaev leg.

DISTRIBUTION. Pakistan (new record). -Tajikistan, Afghanistan.

\section{Amblyptilia sp.}

MATERIAL EXAMINED. Pakistan: Khyber Pakhtunkhwa province, Shogran, $34^{\circ} 38^{\prime} \mathrm{N}$, 73²8' E, h=2300 m, 6-14.VIII 2019, 1 옹. S. Evdoshenko \& V. Siniaev leg.

REMARKS. The species of the genus Amblyptilia were not reported for Pakistan, but the correct identification of a single female is impossible.

\section{Cnaemidophorus rhododactylus (Denis et Schiffermüller, 1775)}

MATERIAL EXAMINED. Pakistan: Khyber Pakhtunkhwa province, near Naran, 34 56 N, 7345' E, h=2600 m, 29.VII 2019, 1 đ̂, S. Evdoshenko \& V. Siniaev leg.

DISTRIBUTION. Pakistan (new record). - Europe, Russia (European part, Siberia, Far East), Caucasus, Turkey, Lebanon, Turkmenistan, Tajikistan, Kazakhstan, Korea, Japan, India, North Africa, North America.

\section{Stenoptilia bipunctidactyla (Scopoli, 1763)}

DISTRIBUTION. Pakistan (Gielis, 2003). - Europe, (Russia European part, Siberia, Far East), Armenia, Turkey, Iran, Kazakhstan, Tunisia, Egypt, Syria, Lebanon.

\section{Stenoptilia madyana Arenberger, 1999}

MATERIAL EXAMINED. Pakistan: Khyber Pakhtunkhwa province, Shogran, 34³8' N, 73²8' E, h=2300 m, 6-14.VIII 2019, 2 đ', S. Evdoshenko \& V. Siniaev leg.

DISTRIBUTION. Pakistan (Arenberger, 1999b). 


\section{Stenoptilia molleti Gibeaux, 1992}

DISTRIBUTION. Pakistan: Baltiatan (Gibeaux, 1992).

\section{Stenoptilia stigmatodactyla (Zeller, 1852)}

MATERIAL EXAMINED. Pakistan: an administrative territory Gilgit Baltistan, Astor River, Chilam, $35^{\circ} 08^{\prime} \mathrm{N}, 75^{\circ} 02^{\prime} \mathrm{E}, \mathrm{h}=3050 \mathrm{~m}, 1-4$.VIII 2019, 1 \%, S. Evdoshenko \& V. Siniaev leg.

DISTRIBUTION. Pakistan (new record). - Europe, Russia (European part, South Siberia, Yakutia, Magadan region), Turkey, Armenia, Iran, Kazakhstan, North Africa.

\section{Procapperia kuldschaensis (Rebel, 1914)}

DISTRIBUTION. Pakistan: Hazara: Kaghan (Arenberger, 2002). - Russia (southern Ural, South Siberia), Turkey, Kazakhstan, Uzbekistan, Kyrgyzstan, Tajikistan, Afghanistan, China, Mongolia.

\section{Crombrugghia distans (Zeller, 1847)}

DISTRIBUTION. Pakistan: Swat, Madyan (Arenberger, 2002). - North Africa, Europe, Turkey, Armenia, Georgia, European part of Russia, Kazakhstan, southern Siberia, Turkmenistan, Kyrgyzstan, Uzbekistan, Tajikistan, Iran, Afghanistan, Nepal, China, India.

\section{Stenodacma pyrrhodes (Meyrick, 1889)}

DISTRIBUTION. Pakistan: Nathia Gali, Hazara Manshera, Thakot, Prov. Swat, Madyan (Arenberger, 2002). - China, Taiwan, Korea, Japan, India, Nepal, Sri Lanka, Thailand, Vietnam, Australia.

\section{Stenodacma wahlbergi (Zeller, 1852)}

DISTRIBUTION. Pakistan: Kohistan: Pattan (Arenberger, 2002). - Saudi Arabia, Yemen, Iran, Southern Africa, Cameroon, Swaziland, Zimbabwe, Tanzania, Kenya, Madagascar, St. Helena Island, Comoros, Reunion Island, Rodriguez Island, Mauritius, Seychelles, Ghana, Uganda.

\section{Megalorhipida leucodactylus (Fabricius, 1794)}

DISTRIBUTION. Pakistan: Karachi, Lyallpur, Rawalpindi, Peshawar, Hangu (Arenberger, 2002). - Widespread throughout tropical and subtropical regions.

\section{Exelastis ebalensis (Rebel, 1907)}

Alucita ebalensis Rebel, 1907: 114 (holotype - Ô, Yemen: Aden, Ma'alle Ebene; deposited in the Museum of Natural History of Vienna).

Karachia xylochromella Amsel, 1968: 15 (holotype - Ô, Pakistan: Karachi; deposited in the Museum of Natural History of Vienna); syn. $\mathbf{n}$.

DISTRIBUTION. Pakistan: Karachi (Amsel, 1968). - Yemen, Oman, Kenya, Ghana.

REMARKS. The images of holotype and figures of male genitalia of Exelastis ebalensis from Yemen given by Arenberger (1999a) and the same given in the original description of Karachia xylochromella from Pakistan (Amsel, 1968) are identical; wherefore new synonymy is proposed here. 


\section{Gypsochares catharotes (Meyrick, 1908)}

DISTRIBUTION. Pakistan (Gielis, 2003). - India, Nepal, Thailand, Southern Africa, Kenya.

Hellinsia pectodactyla (Staudinger, 1859)

MATERIAL EXAMINED. Khyber Pakhtunkhwa province, Shogran, $34^{\circ} 38^{\prime} \mathrm{N}, 73^{\circ} 28^{\prime} \mathrm{E}$, $\mathrm{h}=2300 \mathrm{~m}, 6-14$.VIII 2019, 1 ㅇ, S. Evdoshenko \& V. Siniaev leg.

DISTRIBUTION. Pakistan (new record). - Europe, European part of Russia, Kazakhstan, Kyrgyzstan, Tajikistan, Afghanistan, Pakistan, Nepal, India, Kenya, Canada, USA.

\section{Emmelina monodactyla (Linnaeus, 1758)}

DISTRIBUTION. Pakistan: Swat, Hazara (Arenberger, 1995). - Europe, Russia (South Siberia east to Tuva) Lebanon, Israel, Egypt, Syria, Jordan, Saudi Arabia, Yemen, Turkey, Iraq, Iran, Armenia, Afghanistan, Kazakhstan, Kyrgyzstan, Turkmenistan, Uzbekistan, Tajikistan, India, Philippines, Mongolia, China, Korea, North and South America.

\section{Pterophorus ischnodactyla (Treitschke, 1833)}

DISTRIBUTION. Pakistan: Karachi (Arenberger, 1995). - Europe, Syria, Israel, Lebanon, Oman, Yemen, Bahrain, Turkey, Iraq, Mongolia, Southern Africa.

\section{CONCLUSION}

Currently, 19 species of Pterophoridae are recorded from Pakistan. Undoubtedly, in future the number of species may be increased considerably for the region with such a variety of natural landscapes.

\section{REFERENCES}

Amsel, H.G. 1968. Zur Kenntnis der Microlepidopteren Fauna von Karachi (Pakistan). Stuttgarter Beiträge zur Naturkunde, 191: 14-15.

Arenberger, E. 1995. Pterophoridae I. In: Amsel, H. G., Gregor, F. \& Reisser, H. (Eds), Microlepidoptera Palaearctica 9. Wien. 258 pp. +153 pls.

Arenberger, E. 1999a. Die Pterophoridae des Jemen. Esperiana, 7: 245-262.

Arenberger, E. 1999b. Pterophoridae aus Zentralasien und angrenzenden Territorien. 2. Teil. Quadrifina, 2: 215-226.

Arenberger, E. 2002. Pterophoridae 2. Deuterocopinae, Platyptiliinae: Trichoptilini, Oxyptilini, Tetraschalini. In: Gaedike, R (Ed.). Microlepidoptera Palaearctica. 11. Seiten. 287 pp. + 96 pls.

Gibeaux, C. 1992. Etude des Pterophoridae (27e note). Caractérisation de taxa nouveaux dans le genre Stenoptilia entraînant la création d'une section coprodactyla Stainton. Bulletin de la Société entomologique de France, 96: 463-471.

Gielis, C. 2003. World catalogue of Insects, 4: Pterophoroidea \& Alucitoidea. Apollo Books, Stenstrup. 198 pp. 